\title{
Basal extracellular dopamine in the nucleus accumbens during amphetamine withdrawal: a 'no net flux' microdialysis study
}

\author{
Donita Crippens ${ }^{\mathrm{a}}$, Dianne M. Camp ${ }^{\mathrm{a}}$, Terry E. Robinson ${ }^{\mathrm{a}, \mathrm{b}, *}$ \\ Department of a Pychology and ${ }^{b}$ Neuroscience Program, The University of Michigan, Ann Arbor, MI. USA
}

(Received 17 June 1993; Revised version received 10 August 1993; Accepted 20 September 1993)

Key words: Striatum; Psychomotor stimulant; Drug dependence

\begin{abstract}
The basal extracellular concentration of dopamine in the nucleus accumbens was quantified using the 'no net flux' microdialysis method, in rats undergoing withdrawal from D-amphetamine. Rats were initially pretreated with saline, or an escalating dose amphetamine regimen known to produce a robust withdrawal syndrome, and extracellular dopamine was quantified 3 or 28 days after the last pretreatment injection. There was no effect of amphetamine pretreatment on the basal extracellular concentration of dopamine in the nucleus accumbens, or on the "in vivo recovery" of dopamine, estimated by 'no net flux' microdialysis. It is suggested that amphetamine withdrawal is not necessarily accompanied by changes in the basal extracellular concentration of dopamine in the nucleus accumbens.
\end{abstract}

Following the discontinuation of chronic amphetamine use, humans develop withdrawal symptoms similar to those associated with endogenous depression, including excessive sleep, lethargy, fatigue, and dysphoria [1, 3 . 14]. Rats also show an amphetamine withdrawal syndrome, and the symptoms include behavioral hypoactivity [13] and a decrease in the efficacy of intracranial selfstimulation reward (ICSS) [2]. For example, for at least 7 days following the discontinuation of an escalating dose amphetamine regimen, rats exhibit both a decrease in spontaneous nocturnal locomotion $[7,8]$, and an increase in the threshold required to maintain ICSS [5]. By 28 days of withdrawal, both spontaneous motor activity and ICSS thresholds return to control levels $[5,7]$.

There is considerable interest in the nature of the neuroadaptations underlying the symptoms of drug withdrawal, and recent research has focused on the role of mesotelencephalic dopamine systems. Indeed, it is reported that withdrawal from not only amphetamine, but from a number of addictive drugs, including morphine, alcohol and cocaine, is associated with a decrease in the basal extracellular concentration of dopamine in the ventral striatum $[10,11]$. The purpose of this study was to reexamine the hypothesis that amphetamine withdrawal is associated with a decrease in dopamine neurotransmis-

*Corresponding author. Neuroscience Laboratory Building, The University of Michigan, 1103 E. Huron Street, Ann Arbor, MI 48104 1687, USA. Fax: (1) (313) 936-2690. E-Mail: GB92@um.cc.umich.edu. sion in the ventral striatum, using the 'no net flux' microdialysis method [4].

Drug pretreatment. Male Holtzman rats, initially weighing $300-500 \mathrm{~g}$, were housed individually in wire hanging cages on a 14:10 light/dark cycle (lights on at $07.00 \mathrm{~h}$ ), with free access to food and water. Rats were pretreated with D-amphetamine sulfate (weight of the salt) using an escalating-dose regimen ( 1 to $10 \mathrm{mg} / \mathrm{kg}$, i.p.), in which the drug was administered twice daily, 5 days per week for 6 weeks, as described previously [7]. Control rats received $0.9 \%$ saline, and all injections were given in the home cage.

Surgical and microdialysis procedures. After 4 to 5 weeks of drug pretreatment, and on the first of 2 drugfree days, each rat was anesthetized with sodium pentobarbital and a single 22-gauge stainless steel guide cannula was fixed stereotaxically on the dural surface above the nucleus accumbens. Pretreatment injections resumed 2 days later. Either 2 or 27 days after the final injection, each animal was placed in a $30 \times 50 \mathrm{~cm}$ plexiglass chamber, and a concentric dialysis probe (O.D. $250 \mu \mathrm{m}, 2 \mathrm{~mm}$ effective length) was inserted into the nucleus accumbens via the guide cannula. The probe was perfused with a solution containing $145 \mathrm{mM} \mathrm{NaCl}, 2.7 \mathrm{mM} \mathrm{KCl}, 1.2$ $\mathrm{mM} \mathrm{CaCl}, 1.0 \mathrm{mM} \mathrm{MgCl}$ and $0.2 \mathrm{mM}$ ascorbic acid ( $\mathrm{pH} 7.3$ ) at $0.3 \mu \mathrm{l} / \mathrm{min}$. (See ref. 9 for details concerning the dialysis procedures.) The animals were then left undisturbed overnight. The next morning ( 3 or 28 days after the last injection) the perfusion rate was increased 
to $1.5 \mu \mathrm{l} / \mathrm{min}$ and, after a minimum stabilization period of $1 \mathrm{~h}$, at least four $30 \mathrm{~min}$ samples of dialysate were collected. These samples represent, therefore, a conventional dialysis experiment. The perfusion solution described above, which contained $0 \mathrm{nM}$ of dopamine, was then changed to one containing (in addition to the salts) $2.5,5$ or $10 \mathrm{nM}$ of dopamine in a pseudo-random order, as described by Parsons and Justice [6]. After a $90 \mathrm{~min}$ equilibration period, three additional dialysate samples were collected. (Pilot work revealed that within $90 \mathrm{~min}$ the dialysate concentrations of dopamine were stable; that is, successive samples varied by less than $10 \%$.) This procedure was then repeated for each of the two remaining concentrations of dopamine. Thus, each animal was perfused first with a perfusion solution containing $0 \mathrm{nM}$ of dopamine, followed by solutions containing 2.5,5 and $10 \mathrm{nM}$ of dopamine. Dopamine in dialysate was separated by HPLC and analyzed using electrochemical detection, as described previously [9]. The perfusion solutions containing $0,2.5,5$ and $10 \mathrm{nM}$ of dopamine were used for the chromatographic standards.

All probes were tested for recovery of dopamine in vitro prior to use, and there were no group differences in in vitro recovery, which averaged $( \pm$ S.E.M.) $14.2 \pm 0.94 \%$ for dopamine. In addition, only animals whose probes were histologically verified to have at least $75 \%$ of the dialysis surface within the nucleus accumbens were retained in the experiment.

Fig. 1 depicts the net flux of dopamine across the dialysis membrane for each of the three groups, as a function of the concentration of dopamine in the perfusion solution. The analysis illustrated in Figure 1 provides two important values. The first is the point of 'no net flux'; that is, the concentration of dopamine in the perfusion solution at which dopamine diffusion into the probe equals that out of the probe. This value provides an estimate of the true extracellular concentration of dopamine [6]. It is evident in Figure 1 that there was no change in the point of 'no net flux' of dopamine in association with amphetamine withdrawal. The mean ( \pm S.E.M.) extracellular concentration of dopamine for each group, derived from linear regression analyses, was: saline pretreated, $4.726 \mathrm{nM}( \pm 0.676)$; 3-day amphetamine withdrawal, $5.109 \mathrm{nM}( \pm 0.504)$; 28-day amphetamine withdrawal, $4.716 \mathrm{nM}( \pm 0.107)$.

The second important value derived from Fig. 1 is the slope of the regression line, which represents 'in vivo recovery' [6]. Again, there were no significant group differences. The mean 'in vivo recovery' value for each group was: saline pretreated, $23.7 \pm 3.6 \%$; 3 -day amphetamine withdrawal, $26.1 \pm 4.1 \%$; 28-day amphetamine withdrawal, $24.2 \pm 2.2 \%$. As reported previously [6], recovery in vivo was greater than recovery in vitro.

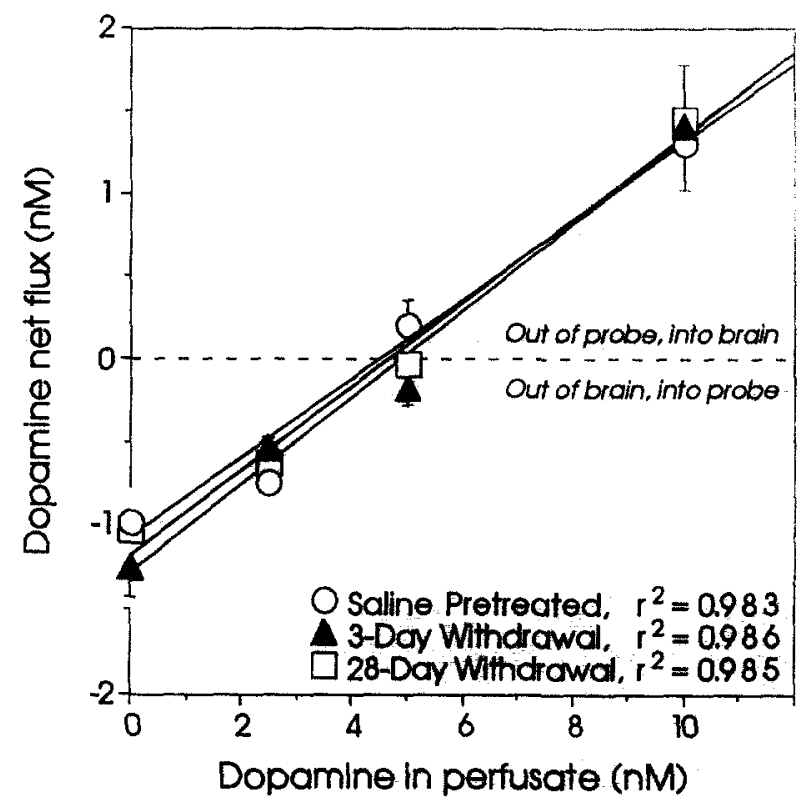

Fig. 1. Dopamine net flux (the concentration of dopamine into the probe minus the concentration of dopamine out of the probe) across the dialysis membrane in the nucleus accumbens, plotted as a function of the concentration of dopamine in the perfusion solution. Symbols represent group means \pm S.E.M. Negative values on the $y$-axis indicate net dopamine diffusion from the brain into the probe, and positive values indicate net diffusion into the brain from the probe. The point of zero flux ('no net flux') for each animal was extrapolated from a linear regression line, and this point represents the concentration of dopamine at which diffusion into the probe equals that out of the probe. This value presumably is equal to the 'true' extracellular concentration of dopamine [6]. There was no significant effect of amphetamine withdrawal on the point of no net flux $\left(F_{2,12}=0.24, P=0.79\right)$. The slope of the regression line indicates the rate of 'in vivo recovery', or efficiency, of the probe [6]. There was no significant effect of amphetamine withdrawal on this measure $\left(F_{2,12}=0.127, P=0.88\right)$. The $r^{2}$ values shown on the figure indicate that for all 3 groups over $98 \%$ of the variance in the data can be attributed to a linear relationship between the net flux of dopamine across the probe membrane, and the concentration of dopamine in the perfusate. The open circles represent values for salinepretreated rats $(n=4)$, the closed triangles values for amphetaminepretreated rats withdrawn for 3 days $(n=6)$, and the open squares values for amphetamine-pretreated rats withdrawn for 28 days $(n=5)$.

Note that the dopamine net flux values for the $0 \mathrm{nM}$ dopamine perfusion solution represent a conventional dialysis experiment. There was also no effect of amphetamine withdrawal when these values were analyzed separately $\left(F_{2,12}=0.869, P=0.44\right)$. In addition, there was no significant effect of amphetamine withdrawal, or the addition of dopamine to the perfusion solution, on the concentration of DOPAC in dialysate (data not shown).

In the present study rats were pretreated with $d$-amphetamine using an aggressive dosing regimen, in which animals received two injections per day, with dose gradually escalating from 1 to $10 \mathrm{mg} / \mathrm{kg}$ over 6 weeks. The discontinuation of this amphetamine pretreatment regimen is associated with a marked withdrawal syndrome 
that lasts for at least 7 days, and consists in part of 'behavioral depression' and changes in motivated behavior $[5,7]$. In the present study, however, amphetamine withdrawal was not associated with any change in the extracellular concentration of dopamine in the nucleus accumbens, or in the 'in vivo recovery' of dopamine, as determined by 'no net flux' microdialysis.

In contrast, using conventional dialysis techniques, Rossetti et al. [10] found that extracellular dopamine is significantly decreased in the ventral striatum for up to 5 days following the discontinuation of amphetamine pretreatment. There are several differences between the present study and that by Rossetti and his colleagues [10] that could potentially account for the discrepancy.

First, different microdialysis methods were used. As mentioned above, Rossetti et al. [10] used conventional dialysis methods, and the 'no net flux' method was used here. This is not a plausible explanation, however, because a conventional dialysis experiment was incorporated into the present experiment, and there was no effect of amphetamine withdrawal on dopamine when these data were analyzed separately. In addition, using conventional microdialysis methods, both Segal and Kuczenski [12] and Wolf et al. [15] report no change in the basal extracellular concentration of dopamine in the nucleus accumbens during the first 5 days of amphetamine withdrawal, consistent with the 'no net flux' data reported here. Furthermore, we have used conventional on-line dialysis methods to sample dopamine across the entire light/dark cycle in animals tested 3,7 or 28 days following discontinuation of the escalating dose amphetamine regimen used here (P. Paulson and $\mathrm{T}$. Robinson. unpublished experiments), and found no effect of amphetamine withdrawal on basal dopamine concentrations in the nucleus accumbens. The discrepancy does not appear to be due, therefore, to differences between conventional and 'no net flux' dialysis methods.

Second, different amphetamine pretreatment regimens were used in the two experiments. One could postulate that the regimen used by Rossetti et al. [10] produced a more pronounced withdrawal syndrome than the regimen used here, but this seems unlikely. In the present study the dose of amphetamine was escalated from 1 to $10 \mathrm{mg} / \mathrm{kg}$ over 6 weeks, for a total of 60 injections (cumulative dose $=356 \mathrm{mg} / \mathrm{kg}$ ), and this regimen is known to produce a marked behavioral withdrawal syndrome lasting for at least seven days $[5,7,8]$. In contrast, Rossetti et al. [10] gave a constant dose of only $1.5 \mathrm{mg} / \mathrm{kg}$ over 16 days, for a total of 32 injections (cumulative dose $=48$ $\mathrm{mg} / \mathrm{kg}$ ). In addition, both Segal and Kuczenski [12] and Wolf et al. [15] used a relatively moderate pretreatment regimen, and as here, found no effect of amphetamine withdrawal on extracellular dopamine.
Another difference in the pretreatment regimens is that Rossetti et al. [10] gave amphetamine every day for 16 successive days, whereas in the present study amphetamine was given in six weekly cycles of 5 successive drug days followed by 2 drug-free days. There were also 2 drug-free days between two 5-day cycles of amphetamine pretreatment in the Wolf et al. [15] experiment. Therefore, in the experiment by Rossetti et al. [10], animals experienced withdrawal for the very first time when undergoing microdialysis. But in the present study animals experienced withdrawal five times prior to the microdialysis test session, and in the Wolf et al. [15] study once previously. It is interesting to speculate, therefore, that the extracellular concentration of dopamine may be affected differently by the first experience with withdrawal than it is by subsequent experiences with withdrawal. This hypothesis is not supported, however, by the Segal and Kuczenski [12] study, in which the dialysis test session took place during the first experience with withdrawal. In addition, the behavioral and psychological symptoms of withdrawal should not dissipate with repeated withdrawal, but should be enhanced. Therefore, even if there were an effect of previous experience with withdrawal, the results reported here would suggest that a decrease in the extracellular concentration of dopamine in the nucleus accumbens is not necessarily associated with symptoms of amphetamine withdrawal.

A third difference between the present study and Rossetti et al. [10] is that different style microdialysis probes were used. Rossetti et al. [10] used horizontal transverse probes that sampled the ventral striatum. whereas in this study concentric probes were placed vertically into the nucleus accumbens. Segal and Kuczenski [12] and Wolf et al. [15] also used concentric-style probes, and Segal and Kuczenski [12] sampled the medial striatum in addition to the nucleus accumbens. Nevertheless, the different probe designs necessarily result in sampling slightly different regions in the ventral striatum. Perhaps more important, however, use of a transverse probe requires a major surgical procedure, including surgical levels of anesthesia, the day before a dialysis test session. In this study no surgery or anesthesia was required at the time of probe implantation, because the probes were simply lowered via a previously implanted guide cannula. It is possible, therefore, that there is an interaction between amphetamine withdrawal and the trauma associated with surgery, which affects basal extracellular dopamine concentrations measured the next day.

In summary, we found that amphetamine withdrawal was not accompanied by changes in the basal extracellular concentration of dopamine in the nucleus accumbens. or in the 'in vivo recovery' of dopamine, as determined by 'no net flux' microdialysis. Although there may be a 
correlation between amphetamine withdrawal and the basal concentration of dopamine in ventral striatal dialysate under some experimental conditions, this study suggests it is not an obligatory relationship [12,15, as well].

\section{Supported by NIDA Grant 04294.}

1 Gawin, F.H. and Ellinwood Jr., E.H., Cocaine and other stimulants. Actions, abuse, and treatment, New Engl. J. Med., 318 (1988) 1173-1182.

2 Kokkinidis, L. and Zacharko, R.M., Response sensitization and depression following long-term amphetamine treatment in a selfstimulation paradigm, Psychopharmacology (Berlin), 68 (1980) 73 76.

3 Kramer, J., Fischman, V. and Littlefield, D., Amphetamine abuse, JAMA, 201 (1967) 305-309.

4 Lönnroth, P., Jansson, P.A. and Smith, U., A microdialysis method allowing characterization of intercellular water space in humans, Am. J. Physiol., 253 (1987) E228-E231.

5 Munn, E.M. and Wise, R.A., The effects of escalating doses of $\mathrm{D}$-amphetamine (AMPH) on lateral hypothalamic intracranial selfstimulation (ICSS), Soc. Neurosci. Abstr., 18 (1992) 364.

6 Parsons, L.H. and Justice Jr., J.B., Extracellular concentration and in vivo recovery in the nucleus accumbens using microdialysis, $J$. Neurochem., 58 (1992).

7 Paulson, P.E., Camp, D.M. and Robinson, T.E., The time course of transient behavioral depression and persistent behavioral sensitization in relation to regional brain monoamine concentrations during amphetamine withdrawal in rats, Psychopharmacology, 103 (1991) 480-492.

8 Robinson, T.E. and Camp, D.M., Long-lasting effects of escalating doses of D-amphetamine on brain monoamines, amphetamine-induced stereotyped behavior and spontaneous nocturnal locomotion, Pharmacol. Biochem. Behav., 26 (1987) 821-827.
9 Robinson, T.E. and Camp, D.M., The feasibility of repeated microdialysis for within-subjects design experiments: studies on the mesostriatal dopamine system, In T.E. Robinson and J.B. Justice Jr. (Eds.), Microdialysis in the Neurosciences, Elsevier, Amsterdam. 1991, pp. 189-234.

10 Rossetti, Z.L., Hmaidan, Y. and Gessa, G.L., Marked inhibition of mesolimbic dopamine release: a common feature of ethanol, morphine, cocaine and amphetamine abstinence in rats, Eur. J. Pharmacol., 221 (1992) 227-234.

11 Rossetti, Z.L., Melis, F., Carboni, S. and Gessa, G.L., Dramatic depletion of mesolimbic extracellular dopamine after withdrawal from morphine, alcohol or cocaine: a common neurochemical substrate for drug dependence. In P.W. Kalivas and H.H. Samson (Eds.), The Neurobiology of Drug and Alcohol Addiction, The New York Academy of Sciences, New York, 1992, pp. 513-516.

12 Segal, D.S. and Kuczenski, R., In vivo microdialysis reveals a diminished amphetamine-induced DA response corresponding to behavioral sensitization produced by repeated amphetamine pretreatment, Brain Res., 571 (1992) 330-337.

13 Utena. H., Behavioral aberrations in methamphetamine-intoxicated animals and chemical correlates in the brain. In T. Tokizane and J.P. Schade (Eds.), Progress in Brain Research, Vol. 21B, Coerrelative Neurosciences: Clinical Studies, Elsevier, Amsterdam, 1966, pp. 192-207.

14 Watson, R., Hartmann, E. and Schildkraut, J.J., Amphetamine withdrawal: affective state, sleep patterns, and MHPG excretion, Am. J. Psychiatry, 129 (1972) 263-269.

15 Wolf, M.E., White, F.J., Nassar, R., Brooderson, R.J. and Khansa, M.R., Differential development of autoreceptor subsensitivity and enhanced dopamine release during amphetamine sensitization, J. Pharmacol. Exp. Ther., 264 (1993) 249-255. 